Cite this: Soft Matter, 2013, 9, 10442

Received 13th March 2013

Accepted 18th July 2013

DOI: $10.1039 / c 3 s m 50722 \mathrm{j}$

www.rsc.org/softmatter

\title{
Physical modelling of the nuclear pore complex
}

\author{
Dino Osmanović, ${ }^{\text {*a }}$ Ariberto Fassati, ${ }^{\mathrm{b}}$ Ian J. Ford ${ }^{\mathrm{a}}$ and Bart W. Hoogenboom ${ }^{\mathrm{a}}$
}

Physically interesting behaviour can arise when soft matter is confined to nanoscale dimensions. A highly relevant biological example of such a phenomenon is the Nuclear Pore Complex (NPC) found perforating the nuclear envelope of eukaryotic cells. In the central conduit of the NPC, of $\sim 30-60 \mathrm{~nm}$ diameter, a disordered network of proteins regulates all macromolecular transport between the nucleus and the cytoplasm. In spite of a wealth of experimental data, the selectivity barrier of the NPC has yet to be explained fully. Experimental and theoretical approaches are complicated by the disordered and heterogeneous nature of the NPC conduit. Modelling approaches have focused on the behaviour of the partially unfolded protein domains in the confined geometry of the NPC conduit, and have demonstrated that within the range of parameters thought relevant for the NPC, widely varying behaviour can be observed. In this review, we summarise recent efforts to physically model the NPC barrier and function. We illustrate how attempts to understand NPC barrier function have employed many different modelling techniques, each of which have contributed to our understanding of the NPC.

\section{Introduction}

Confinement in soft matter has important implications in biological contexts, for example in the Nuclear Pore Complex (NPC), where unstructured proteins are confined within a roughly cylindrical channel. They are able to perform a highly delicate gating function.

${ }^{a}$ London Centre for Nanotechnology and Department of Physics and Astronomy, University College London, Gower Street,WC1E 6BT, UK. E-mail: d.osmanovic@ucl. ac.uk

${ }^{b}$ Wohl Virion Centre and MRC Centre for Medical Molecular Virology, University College London, Gower Street, WC1E 6BT, UK
All eukaryotic cells are subdivided into a nucleus and a cytoplasm. These two compartments of the cell are separated by the nuclear envelope, a double membrane which surrounds the nucleus. Permeating the nuclear envelope is the nuclear pore complex. The NPC is the main path for translocation of macromolecules between the nucleus and the cytoplasm. It is one of the largest protein complexes within the cell. Its structure and function has been subject to considerable experimental work. ${ }^{1}$ The various methods employed, such as cryoelectron tomography, co-immunoprecipitation, fractionation, X-ray crystallography and structural modelling, ${ }^{3,4}$ have given us an understanding of the structure of the NPC.

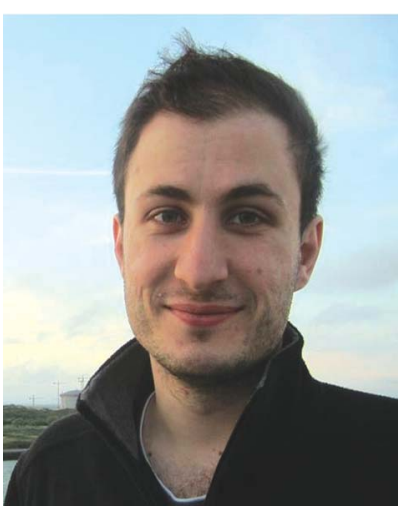

Dino Osmanovic is currently studying for a PhD in the modelling of Nuclear Pore Complexes at the London Centre for Nanotechnology. He received an $\mathrm{MSci}$ in Physics from University College London in 2011.

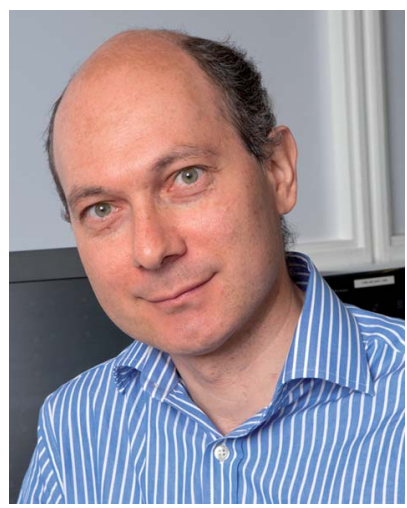

Ariberto Fassati graduated in Medicine at the University of Milan, Italy, in 1991. He obtained a PhD from UMDS Guys Hospital, London, was awarded a Wellcome Trust International Fellowship with Steve Goff at Columbia University, New York, a Wellcome Trust Career Development Fellowship in 2000 with Robin Weiss at University College London and a Wellcome Trust University Award in 2004. He is a Reader (tenured Associate Professor) in Cellular and Molecular Virology and the Director of the Wohl Virion Centre at UCL. Fassati works on host-cofactors for virus infection, viral nuclear import, and transmissible cancers. 
A schematic of the NPC is shown in Fig. 1A. It has an eightfold symmetry around the central axis, having an outer diameter of $90-120 \mathrm{~nm}$, a height of $30-50 \mathrm{~nm}$ and consisting of multiple copies of about 30 different types of proteins, called nucleoporins or nups. There are distinct classes of nups; some are structural and constitute the NPC scaffold, others are nonstructural, called phenylalanine-glycine repeat containing nups (FG nups). FG nups contain long natively disordered protein regions, which are enriched by Phe-Gly dipeptides. In addition to this, the FG nups contain structured domains that anchor them to the structural nups. The structural nups form a cytoplasmic and a nuclear ring and delimit a central channel, which is filled by the unfolded FG domains. Transport of macromolecules is via the central conduit of $\sim 30-60 \mathrm{~nm}$ diameter, which contains a selective barrier. ${ }^{5}$ The barrier allows passive diffusion of solutes less than $5 \mathrm{~nm}$ in diameter, but prevents passage of inert molecules larger than $9 \mathrm{~nm}$ in diameter unless they are chaperoned by nuclear transport receptors (NTRs). ${ }^{6}$ All macromolecules (cargoes) that are transported through the pore contain a nuclear localization signal or a nuclear export signal that binds to a NTR and the NTR bound cargo can then diffuse through the pore. NTRs can be classified as a importin if they bind to nuclear localization signals or exportin if they bind to nuclear export signals.

Transport through the NPC is directional such that many cargoes are only imported into or exported from the nucleus, although other cargoes do shuttle in and out continuously. Directionality of transport is mediated by RanGTP gradient across the nuclear envelope. The small GTPase Ran, in its GTP bound form, induces dissociation of cargoes with a nuclear localization signal from the NTRs. Conversely, it promotes association of cargoes with an nuclear export signal to the NTRs. NTRs, on the other hand, shuttle freely between nucleus and cytoplasm. ${ }^{6}$ The RanGTP gradient, high in the nucleus and low in the cytoplasm, ensures that association and dissociation of NTRs and their cargoes happens in the right place and in a directional way. ${ }^{7,8}$

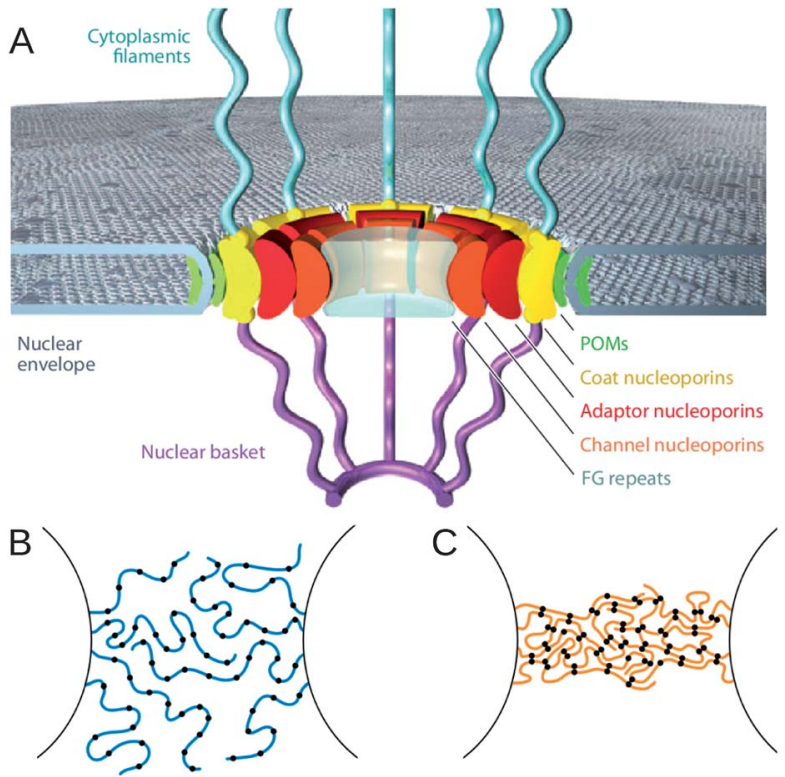

Fig. 1 (A) Simplified representation of the NPC, showing approximate locations of nups within the pore. The disordered FG nups extend through the pore and collectively make up the permeability barrier. Reproduced with permission from Hoelz et al. (2011) (CAnnual Reviews. ${ }^{1}$ (B and C) Show different proposed models for the nature of this barrier. (B) Has more freely fluctuating polymers, as in the virtual gate model, which would maintain a permeability barrier through entropic exclusion. (C) Is the selective phase model, where the polymers interact to form a highly crosslinked gel, forming an energetic barrier to transport. Reproduced from Hülsmann et al. (2012) with permission. Copyright (e)lsevier (2012). ${ }^{2}$

Transport of cargoes through the NPC critically depends on the intermolecular interactions between NTRs and nups. NTRs bind to the FG domains of nups via hydrophobic interactions, which must be finely calibrated to allow translocation and final detachment. $^{9-12}$ The complexity of such interactions is further highlighted by the co-existence of different nups with different properties in the central channel. ${ }^{5,13}$ Yet their overall

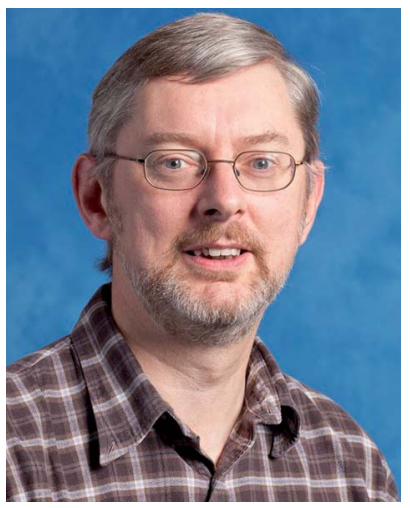

Ian Ford has interests in several branches of statistical physics, in particular the study of nucleation phenomena and kinetic processes in general. He has degrees in Theoretical Physics from Cambridge and oxford Universities and worked for a period at the Harwell Laboratory of the UK Atomic Energy Authority before moving to UCL in 1996. His main contributions have been in aerosol physics, but more recently he has worked on biophysical applications of theories of liquid and polymers, and on entropy generation in nonequilibrium processes. His recently published book 'Statistical physics: an entropic approach' is an attempt to demystify irreversible thermodynamics.

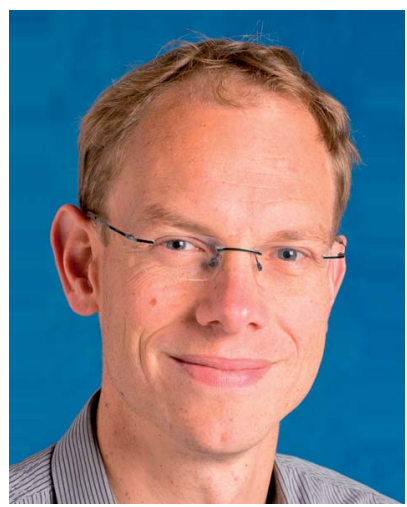

Dr Bart W. Hoogenboom was trained as a physicist at the University of Groningen and the University of Geneva, where he obtained a PhD on scanning tunnelling microscopy and spectroscopy of high-temperature superconductors. During postdoctoral research at the University of Basel, he developed novel atomic force microscopy techniques for imaging biological samples in aqueous environment, working in physics and structural-biology laboratories. Since the end of 2007, he has been a Lecturer at the London Centre for Nanotechnology and the Department of Physics and Astronomy, University College London, where he leads a nano-scale biophysics research group. 
organisation and properties are imperative for the functioning of the permeability barrier, which has a remarkable efficiency allowing 1000 translocation events per second per $\mathrm{NPC}^{\mathbf{1 4}}$ and passage of very large complexes, including viruses, ${ }^{15}$ up to the size of baculovirus with dimensions $30-60 \mathrm{~nm}$ in diameter and a length of 250-300 nm. ${ }^{16}$ Translocation of a small NTR bound cargo through the barrier is also fast, taking approximately 10 milliseconds. ${ }^{17,18}$

The transport mechanism is generally thought to be strongly dependent on hydrophobic interactions between FG-repeats on the nups and the NTRs. ${ }^{19}$ It has also been found that the depletion of certain, more cohesive nups causes the NPC to lose its functionality. ${ }^{2}$ To understand the transport mechanism, it is paramount to know the arrangement of the nups that make up the $\sim 30-60 \mathrm{~nm}$ diameter central channel. These nups are natively unfolded and intrinsically disordered, however, greatly complicating experimental approaches to study their structure. For example, there is experimental evidence for a "central plug" or "central transporter" in the centre of the channel, but it is still unclear whether this plug is caused by the FG nups themselves or by cargoes passing through the pore. The behaviour of FG-nups in the NPC central channel thus represents a still poorly understood example of confined matter, which is literally vital for the cell (non-functional NPCs can lead to cell death $\left.{ }^{9}\right)$.

\section{Qualitative models of the NPC}

Several simplified and often conflicting paradigms of the NPC barrier have been suggested. The proposed nature of the permeability barrier is dependent on nup cohesiveness. There has been debate about whether the barrier facing inert (i.e. not bound to a NTR) cargoes is mainly entropic or energetic in nature. The virtual gate model $^{20}$ (see Fig. 1B) suggests that the barrier is entropic. In this model the nups within the central channel of the pore are mainly freely fluctuating and noncohesive, such that any non-NTR bound cargo would be subject to frequent impacts with the nups. NTRs in NTR bound cargo can bind to the FG repeat regions of the nups, thus paying the entropic cost and allowing transport.

At the other end of the spectrum, the selective phase model, ${ }^{\mathbf{1 4}}$ (see Fig. 1C) posits that the barrier is mainly energetic. The FG nups within the pore bind strongly to one another, forming a dense gel across the NPC. NTR bound cargoes can pass through by the binding of those NTRs to the FG repeats, with a greater affinity than the FG repeats bind to one another, thereby reversibly dissolving the gel and allowing transport of larger cargoes.

The reduction of dimensionality model $^{\mathbf{2 1}}$ for transport supposes that the FG repeats form a film on the circumference of the channel, such that NTR bound cargoes slide across this surface, in effect reducing the dimensionality of the random walk, thus increasing translocation rates for the cargoes. This model postulates that there are also some freely fluctuating peptides in the central channel, meaning non-NTR bound cargoes are kept out by entropic exclusion in the same manner as the virtual gate model.
Models such as these are phenomenological approaches to the question of NPC barrier organisation, and their predictions are based upon assumptions about the way that FG nups interact with one another. One would prefer to make more quantitative models of NPC function.

Whilst a lot of experimental work has been done on the nature of the NPC, theoretical modelling has so far made a more modest contribution towards our understanding of the NPC. Problems faced in the modelling of the NPC include the complexity of the intermolecular interactions, the full form of which remains unknown. Additionally, the large number $(\sim 600)$ of proteins within the NPC make a full computational treatment very difficult. In order to model the NPC, simplifying assumptions are usually applied. For instance, it is convenient to treat the NPC as a cylindrical channel with a ring of polymers representing the FG nups anchored around the circumference of the cylinder.

The methods used to model the NPC can be broadly split into a few categories, in the "jigsaw puzzle" approach one attempts to incorporate the detailed structure of all individual nups, while neglecting the possibility of significant structural rearrangement due to intermolecular interactions. In the "diffusion equation" approach most structural detail is neglected while focusing on the general characteristics of transport. Other approaches ("molecular dynamics" and "equilibrium calculations") include explicit macromolecular arrangements and intermolecular interactions, generally requiring significant simplification of the nups under study, and/or the geometry of the system.

Here we will review the various approaches. We note that there is significant overlap between modelling of the NPC and modelling any polymer-grafted pore, ${ }^{22}$ with results obtained in one field having general significance in the other. Polymergrafted pores have possible technological applications to, among others, DNA sequencing or microfluidic control devices. ${ }^{23}$

\section{Jigsaw puzzle approach}

One approach to determining the configuration of nups within the pore is to attempt to fit together the nucleoporins from their partially known experimental structures and affinities. ${ }^{24}$ The experimental data for such calculations can come from a wide variety of sources such as electron microscopy or sedimentation analysis. Combining all the experimental data using specialised algorithms can give an account of the structure of the NPC. This model predicts a core scaffold, a structured network of nucleoporins coating the channel wall of the NPC. Extending from this core scaffold are the disordered FG nups. The FG nups do not extend all the way to the centre of the channel, thus according to this approach the central plug structure seen in experiments would not be caused by the constituent proteins of the NPC. These FG nups provide an effective reduction in diameter of the NPC, allowing diffusive transport of cargoes smaller than the effective pore diameter. Specificity of transport is caused by entropic exclusion.

Other examples of this type of method can be found in the paper by Yamada et al. ${ }^{25}$ Again, by combining the results of 
single-nup experiments and single nup simulations, they attempt to reconstitute the NPC. Different varieties of nups were identified by this approach. There exist nups which adopt extended coil configurations and those that adopt, at least partially, more globular shapes. One may also find hybrids of these two. The model which fits together all of these nups is described as the "forest" model (see Fig. 2). Amongst the predictions of this model is that there exist multiple transport channels for cargoes. It is suggested that the NPC is a chemically heterogeneous, partially organised structure. This is in contrast to the random coil nature of the nups suggested by the other models of NPC transport.

These methods do not model interactions between the different nucleoporins comprehensively so it is unknown whether a full treatment of interactions would result in a differing structure. The dynamics of such models are also difficult to gauge, especially if the nucleoporins undergo significant restructuring to accommodate NTR bound cargoes. Nevertheless they can give a general overview of likely arrangements of the nups within the NPC.

\section{Diffusion equations}

Perhaps the simplest approach to understand the NPC is to model the entire process of transport as a diffusive process. ${ }^{26-30}$ Such problems bear similarity to evaporation problems, ${ }^{\mathbf{3 1}}$ where a particle, in this case a cargo, must ascend over a free energy barrier. The general form of the free energy barrier can be

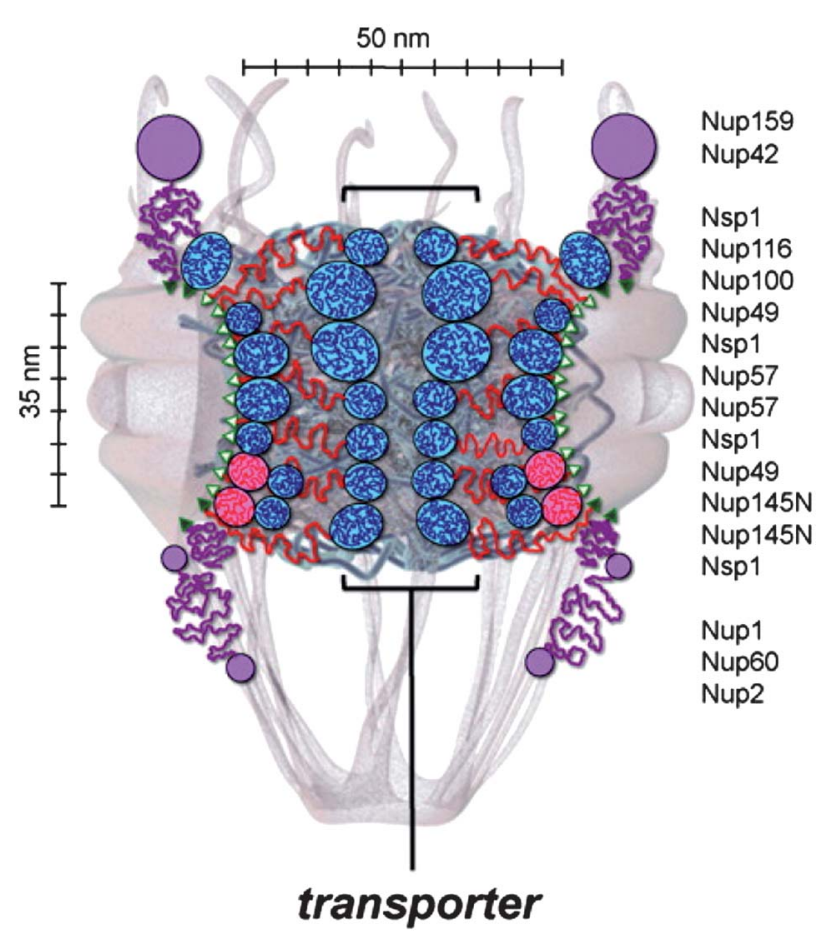

Fig. 2 An overview of the likely positions for different types of nups within the NPC, as derived from a "jigsaw puzzle approach". The nups have a collapsed/ extended dichotomy. The extended nups would be responsible for the "central plug" or "central transporter" mentioned previously. Reproduced from Yamada et al. (2010) ${ }^{25}$ with permission. Copyright (C2010, The American Society for Biochemistry and Molecular Biology. estimated using simple physical arguments or calculated using more advanced methods such as Monte Carlo.

These models can be solved numerically without too much difficulty, allowing us to look at transport rates over a range of parameters such as cargo size and interactions between NTRcargo complex and the nups. For instance, such calculations can confirm the intuitive idea that there exists an optimal binding between the nups and the NTR-cargo complex for greatest transport efficiency. ${ }^{27,28}$ If the interactions were absent then the cargo-NTR complex would not enter the pore. If they were too strong, however, then once the NTR-cargo complex has bound to an FG repeat it would not dissociate and the cargo would be stuck in the pore, blocking transport of other cargoes.

This can be extended further to attempt to understand the selectivity barrier of the NPC. For example, it has been suggested that the barrier is enhanced by the competition between NTR bound cargoes and inert cargoes. ${ }^{29}$ Due to the large rate of transport of cargoes through the NPC, any macromolecular cargo which enters the pore will likely find another cargo in the process of being transported ahead of it. Non-NTR bound cargoes do not have long wait times in the pore, due to lack of interactions between the nups and the NTR. Such an inert cargo is thus more likely to be ejected from the pore through diffusion before the cargo already within the pore has translocated. Due to the interactions between the NTR and the nup, an NTR bound cargo will reside longer in the pore, making it more likely that it will still be within the pore once the other cargo has translocated. In this way the permeability barrier of the pore can be amplified just by the presence of other cargoes.

Diffusion equation modelling of the pore can also give a useful starting point for more elaborate calculations.

\section{Molecular dynamics}

Large-scale Molecular Dynamics (MD) simulations are now the norm in research into protein structure. ${ }^{32}$ This approach is based on solving the equations of motion for every particle in the system. For example, MD can be used to study how proteins fold into their three-dimensional structures. By calculating all the pairwise interactions of the particles, these methods are amongst the most accurate ways of determining structures of large ensembles of interacting particles, and additionally the approach allows us to look at the evolution of structures for short time-scales, though the computational cost of such simulations scales rapidly with the complexity of the system and the process time. A difficulty facing modelling the NPC in such a way is that there about 600 proteins within the pore, making full molecular dynamics simulations of the NPC unfeasible.

One of two approaches is then typical: either one can model a smaller system with full detail ${ }^{33}$ and attempt to infer the full structure from this subsystem, or one can coarse-grain the nups to make molecular dynamics more tractable. ${ }^{34,35}$

Due to the computational cost associated with these approaches, the first studies in this field were obliged to use both assumptions. ${ }^{36}$ These early approaches dispense with most of the detail of the pore in an attempt to gain a qualitative account of transport. Despite their simplicity, these simulations 
give a useful way to test the validity of the different models of barrier organisation. By assuming a priori that the centre of the pore is blocked by a network of FG nups, one can test whether the physical mechanisms proposed by such models are realistic. The mesh size of the network gives a size limit for passive diffusion. Particles larger than the mesh size can diffuse through as long as they are bound to an NTR. As postulated by the selective phase model, importin bound cargoes cause local breakage of the network, thus allowing diffusion of cargoes through the mesh.

When attempting to model a coarse-grained NPC with MD, the form of the interaction can be chosen to represent hydrophobic interactions, which are known to be important among the FG nups. The following form, with some variations, can be used to represent short range repulsion and attractive (e.g. hydrophobic) interactions: $:^{37}$

$$
\phi(\mathbf{r})= \begin{cases}\infty & |\mathbf{r}|<d \\ \varepsilon \exp (-(|\mathbf{r}|-d) / \sigma) & |\mathbf{r}| \geq d\end{cases}
$$

The parameter $\varepsilon$ is usually negative, accounting for the fact that the FG repeats are attractive to one another. ${ }^{38} d$ is the diameter of the part of the nup under consideration, usually taken to be spherical for simplicity and $\sigma$ gives the length scale of the interaction, which is not thought to be more than $1 \mathrm{~nm}$.

The NPC contains many different types of nups, with hydrophilic and hydrophobic domains. Choosing this form of the interaction is implicitly assuming that hydrophobic interactions are the most important interactions in the NPC, though not without experimental justification..$^{\mathbf{3 9 0}}$

The resultant structure of the nups depends upon the parameters chosen to represent the system. MD simulations of cylindrically confined tethered polymers show interesting phase behaviour. Depending on the mutual polymer interaction, one of two regimes is possible. The polymers can swell away from their tethering points, thus blocking the channel, or can collapse back to their tethering points, leaving the centre open. The difference in permeability of these states can be as much as an order of magnitude. ${ }^{\mathbf{1 1}}$

These simulations have given support to differing models for transport. In MD simulations, one introduces inert and NTR bound cargoes on one side of the pore and then measures the rate at which these particles translocate to the other side. The work performed by Mincer and Simon ${ }^{34}$ was amongst the first to attempt to treat the full pore. A cylindrical pore is modelled with FG nups bound to the outer edge. In this treatment the nups are treated as flexible filaments where the flexibility is controlled through a parameter defined at the start of the simulation. The model treats interactions between the FG nups in an approximate way. Collision between FG nups can cause the FG nups to bind to one another, after binding they may dissociate through a user-defined FG-FG off-rate. The interactions between the FG repeats and NTR cargoes are treated in the same way. Significantly, by varying the FG-FG off rate one may observe polymer dynamics corresponding to the different models discussed previously, such as the virtual gate model or the selective phase model. By analysing single particle trajectories through the pore the authors propose a mechanism for the selectivity barrier of the NPC. First the NTR bound cargo diffuses toward the FG nups, whereupon an FG repeat region will bind to it. Other FG-nups will then reorganize around it, binding to the other binding sites of the NTR. Once in this state the NTR bound cargo it fluctuates thermally in position on the cytoplasmic side until it passes through to the nucleoplasmic side. If the concentration of RanGTP (represented in this model as the FG-NTR dissociation rate) is sufficiently high, the NTR bound cargo is released from the FG nups and diffuses into the nucleus.

This model is known as a Brownian ratchet model of transport, where thermal fluctuations play a key role in the transport of NTR bound cargoes. It is claimed that the NTR bound cargo remains bound to the same FG nup during the entire translocation process. The rate limiting step for transport is found to be the reorganization of the FG nups around the NTR bound cargo. Thus the most efficient transport occurs when FG-FG interactions are weak, as this means the FG nups can more easily engulf the NTR bound cargo. Crosslinking of FG nups is not necessary to exclude non-NTR bound cargoes, as entropic exclusion is enough to keep these cargoes from translocating. This model can successfully reproduce some features of NPC transport, such as translocation times and size cut-off.

A similar approach by Moussavi et al., but with a more realistic treatment of the interactions ${ }^{35}$ using a form similar to eqn (1) can develop the picture even more. A bead-spring model of the FG nups is utilised. The FG repeats interact with each other with an $\varepsilon$ of $-3.0 k_{\mathrm{B}} T$ and $\sigma=1.0 \mathrm{~nm}$ with a persistence length of $0.43 \mathrm{~nm}$. The FG nups were found to collapse onto the channel wall, forming a dense gel of FG repeat regions (as in

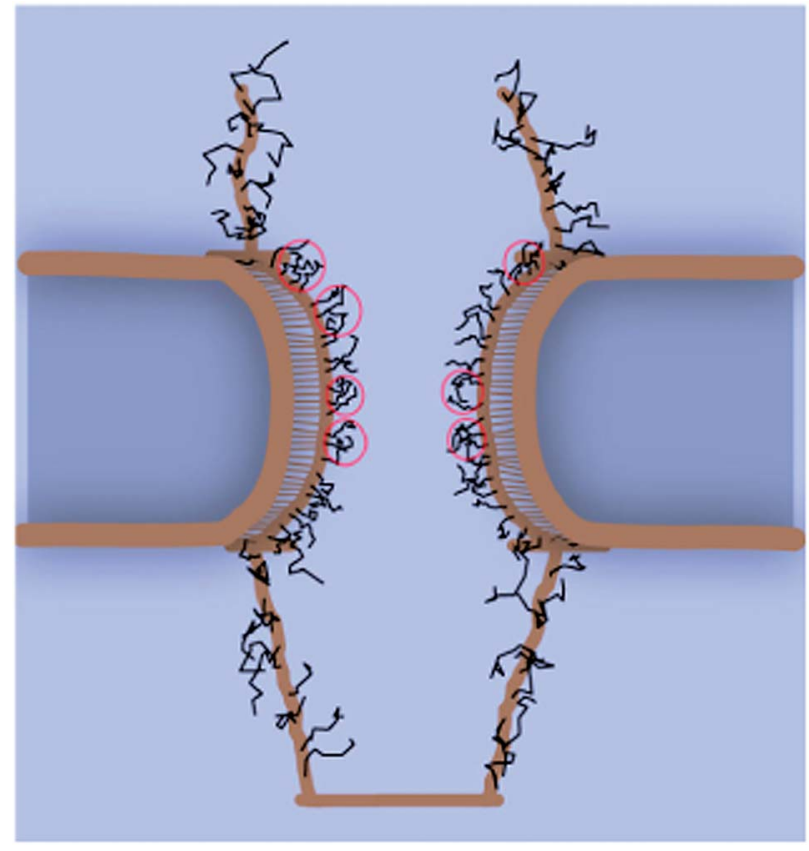

Fig. 3 Snapshot from a equilibrated MD simulation of a simplified model of nups in the pore. The black lines represent the nups, in a conformation near the walls of the channel, Reproduced from Moussavi et al. (2011) $)^{35}$ with permission, Copyright (c)2011, Elsevier. 
Fig. 3). The NTR bound cargo remains bound to FG nups for the majority of its translocation, though not necessarily the same one. During translocation cargoes slide across this gel, in the manner suggested by the reduction of dimensionality model. Calculations of translocation times of cargoes in these models can lead to order of magnitude estimates that compare favourably with experiment, even though the pore channel appears remarkably open, in contrast to experimentally observed structures such as the central plug mentioned earlier.

Moussavi et al. also explored the dependence of transport time on the total size of the NTR-cargo complex. Interestingly, the dependence is non-linear. Up to a diameter of $15 \mathrm{~nm}$, the total translocation time is independent of cargo-NTR complex size. However above this limit, the time increases steeply.

The scarcity of studies done on such systems makes drawing general trends difficult. Further studies exploring different physiologically relevant parameters might yield general trends that could give insight into the NPC barrier. These studies of NPC transport by Mincer et al. and Moussavi et al. yield differing results, illustrating the difficulty of drawing firm conclusions on the basis of modelling, due to the fact that the manner in which the model is constructed can greatly influence the results. As many aspects of FG nups in vivo are unknown, it is important to consider different parameters when conducting computational modelling of NPC transport.

The other possibility, namely modelling just a few nups fully and then attempt to infer NPC function from the physical data of such simulations, has yielded impressive results in determining possible structures as well as effective interactions amongst nups and NTRs. These simulations can reproduce the experimentally known locations of FG binding sites on the NTR surface, ${ }^{\mathbf{4 2 - 4 4}}$ as well as suggesting new binding locations. Interestingly such studies suggest that the viability of transport does not depend on the number of binding sites on the NTR surface but their density.

Atomistic modelling of the nups yields the most accurate representations of nup dynamics. Atomistic simulations performed by Miao and Schulten of 25 nsp1 nucleoporins, grafted on a $5 \times 5$ planar grid, ${ }^{33}$ show the formation of structures similar to polymer brushes. The radius of gyration of a disordered protein within this brush is much larger than the corresponding radius of gyration of a single nsp1 protein. This provides evidence that interactions between the nups could be important for overall nup structure. When an NTR is introduced to this structure ${ }^{45}$ it is found to enter into the brush-like structure, by binding to the FG repeat regions. In contrast an inert molecule was not found to enter the brush structure to the same extent. When such a simulation was repeated without FG repeats, the NTR did not enter the brush-like structure, emphasising the key role of NTR-FG dipeptide interactions in the diffusion of molecules through the brush-like structure.

In this study, FG repeat regions were found to bind to at least ten specific sites on the NTR surface. Interestingly, temporary binding/unbinding of FG-NTR bonds can be observed as the NTR diffuses through the FG nups. However, in all the simulations performed a long lived FG-NTR bond played a key role in allowing penetration of NTRs into the brush. Notably, electrostatic interactions between charged residues on the NTR surface and on the FG nups were found to be significant.

Molecular dynamics simulations are an accurate way of determining structure and dynamics. However, the computational cost associated with MD makes it difficult to draw wide ranging conclusions about NPC transport, as assumptions involved in setting up the models can lead to divergent physical mechanisms of transport. It should be expected that as computers improve more of these assumptions could be relaxed, leading to less ambiguous conclusions. For now, however, MD does show quantitatively the key role that NTR-FG interactions have in the translocation of molecular cargoes. All MD simulations of transport have the common theme that inert cargoes will be rejected, though the precise nature of the barrier is still a matter of discussion.

Traditional methods of molecular dynamics studies largely seem to be sufficient for establishing the equilibrium structure of components of the NPC, but more advanced methods such as parallel tempering ${ }^{47}$ might have a role to play in ensuring that configuration space is fully explored. For the study of relaxational events, such as the overcoming of barriers relevant to transport phenomena, various techniques such as metadynamics, ${ }^{48}$ where ordinary interactions are supplemented by carefully specified history-dependent forces, might allow the potential of mean force for an NTR to be characterised, with or without bound cargo.

\section{Equilibrium calculations}

In addition to these methods one can also use mean field theories such as density functional theory or self-consistent field theory to model the pore. It is difficult to capture full molecular detail using these methods, so one would work with the coarsegrained system already described in the molecular dynamics section. In density functional theory, instead of explicitly calculating all the intermolecular interactions, one represents the particles in the system as a number density function. Using well established physical arguments ${ }^{49}$ one can then construct a free energy functional of the system that depends upon the number density function. This functional is then optimised so that the form of the number density function is such that the free energy is at a minimum. This is then the best approximation to the true density of the system. Self-consistent field theory is similar, in which the system is described by one or more fields, and the field calculated by assuming that one configuration of the fields dominates in the properties of the system.

The advantage of these methods is generally lower computational cost, which can be reduced even further due to the fact that symmetry assumptions are easier to apply. The accuracy usually compares very well to the more accurate MD methods except under certain special conditions such as very strong interactions or when the system is near a phase transition. These methods will also give a free energy of the system, allowing phase maps of likely structures for different parameters to be plotted.

The morphologies of polymers bound within a cylindrical pore can take different forms. Several systematic studies have 
A) good solvent $(\chi=0 \mathrm{kT})$

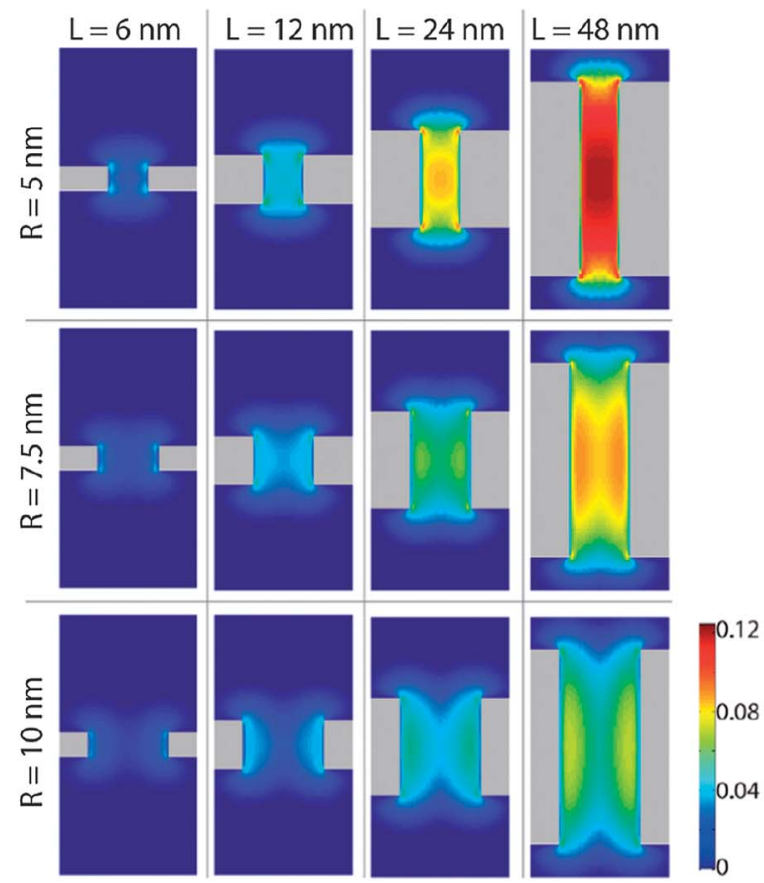

B) poor solvent $(\chi=1.1 \mathrm{kT})$

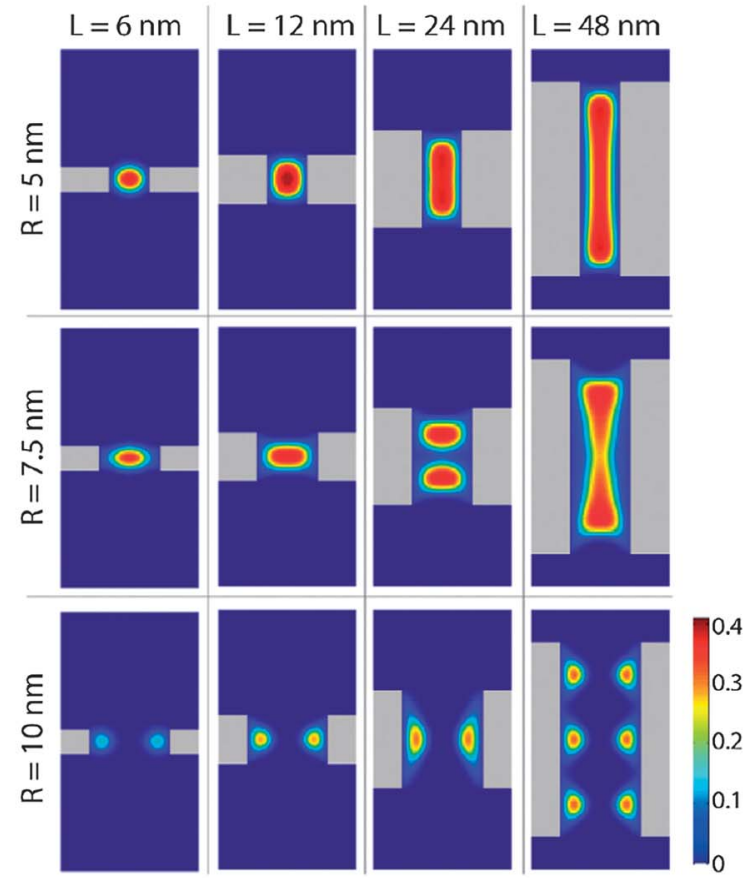

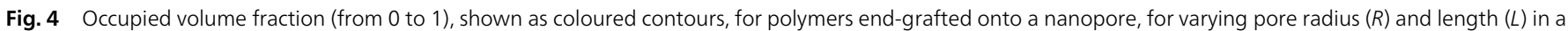
good and poor solvent, reproduced from Peleg et al. (2011) ${ }^{\mathbf{4 6}}$ with permission. Copyright @2011, American Chemical Society.

been performed ${ }^{46,50}$ exploring the parameter space of such a system (see Fig. 4). A common assumptions in mean field calculations of this sort are that the density of the polymers is independent of the azimuthal angle.

Instead of using mean fields, Monte Carlo (MC) methods attempts to gain insight into the ensemble average of certain quantities, such as the density, by using random moves weighted by the energy change of the move..$^{51}$ When conducting MC simulations of the same system, ${ }^{50,52,53}$ however, the assumption that the density is independent of azimuthal angle can be relaxed, showing a wealth of phases that are interesting, not only from a biological perspective, but from a physical one.

These techniques allow us to see more deeply the role of confinement in the conformations of these disordered proteins. Ordinary polymers in bulk solutions are entropic springs, the entropy of stretched polymers goes down in proportion to the square of their extension. When such polymers are tethered on a planar surface, increasing the attraction between the polymer beads results in their drawing closer to their tethering points, as this is both energetically and entropically favourable. However, when polymers are tethered onto a closed surface, such as the inside of a cylinder, increasing the attraction can make it favourable for the polymers to extend away from their tethering points towards the centre, in a manner reminiscent of the pore filling hydrogel of the selective phase model. The entropic cost of extending towards the centre can be paid with the enthalpic gain arising from the fact that on average the polymer beads will be nearer to one another. This would not occur if the polymer attraction were not sufficiently high, in which case a familiar collapse to the tethering points would be observed. Such an effect would not be seen were the polymers anchored on a planar surface. One can also think of these same interactions in terms of solvent quality, i.e. hydrophobicity of the polymers. Decreasing the quality of the solvent, thus increasing the propensity of the hydrophobic polymers to draw together, would lead to formation of a "central plug" like structure, as long as the polymers are long enough to stretch that far.

This physics is explained quantitatively in Fig. 5 , which shows the most likely positions for polymers within a cylindrical nanopore in terms of the interaction parameters between the polymers, for a parameter range that is realistic for FG nups in the NPC central channel. Interestingly, this is the range over which drastic rearrangements of polymers occur. All the hypothetical models described in the introduction can be viewed as regions on this phase diagram. In general, for certain parameters such as length, persistence length and interaction between nups, there is behaviour corresponding to the one of the models described. For example, nups of large persistence length and low interactions will correspond to the virtual gate model, whereas the same but with strong interactions would resemble the selective phase model.

In general one can find simple scaling relations for the radius of gyration of homopolymers tethered within a cylindrical geometry, in terms of parameters such as cylinder length, radius, grafting density and length of polymers. The polymers will exist in different scaling regimes depending on the parameters, where a regime refers to the exponent of the power law within the scaling relation. Changing these parameters can lead to crossover between different regimes. ${ }^{54}$

Polymers can also extend away from their tethering points due to excluded volume interactions between the polymer 

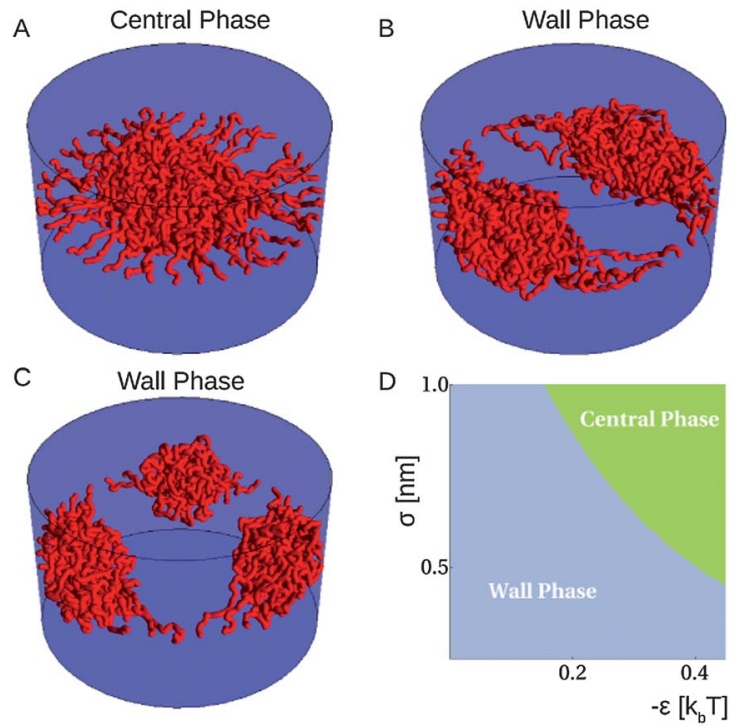

Fig. 5 Monte Carlo simulation results $(A-C)$ corresponding to central and wall phases for the same parameters. One may also observe a further distinction of wall phases, in terms of the number of clumps at the wall corresponding to different (meta)stable configurations. Also shown is a phase map (D) of likely conformations of polymers tethered in a cylindrical geometry will reside, for the $\varepsilon$ and $\sigma$ defined by eqn (1), in a pore of radius $25 \mathrm{~nm}$, with 40 nups of length $100 \mathrm{~nm}$ and segment length $1 \mathrm{~nm}$. As one increases the strength of the attractive interactions between the nups, there is a greater likelihood for polymers to be found in the centre of the pore (green), whereas for weaker interactions the polymers are more likely to be found near the wall of the pore (blue). Adapted from Osmanovic et al. (2012).50

beads. This will generally force the polymers to swell due to crowding. When one explicitly includes the effect of a reservoir above and below the cylinder, the polymers can be shown to stretch out of the pore and into the reservoir. Not only does this decrease the effect of the repulsive interactions, but the conformational entropy is also increased as there are more states available for the polymers outside the pore. In general increasing the strength of attractive interactions will lead to more inhomogeneities of the FG nups within the pore. ${ }^{46}$

Introducing other components into this system, such as free (untethered) polymers, ${ }^{55}$ can be viewed as a simple representation of cargoes interacting with FG nups. As the radius of the pore is decreased one can observe that a free chain penetrates more into the tethered polymer layer, even in the absence of interactions. It was seen that the extent of the overlap between free chains and tethered chains changes non-monotonically. Above a certain radius of the pore the overlap decreases rapidly. However, the significance of this to transport is hard to gauge.

Mean field theories can give quantitatively similar results to more accurate explicitly interacting models at a fraction of the overall computational cost. So long as one uses these theories within their realms of validity, the outputs of such theories give a useful way to determine structures. However, such theories will not be able to capture the molecular detail in the same way as molecular dynamics could. In addition, though dynamical mean field theories exist, application to the NPC has not been attempted. Most modelling of the NPC with mean field theories is restricted to stationary structures of polymer-coated pores, with results that can possibly inform the discussion of NPC function.

Monte Carlo methods have the same problem. As with the mean field theories, dynamical Monte Carlo schemes exist, but to attempt to discern the dynamics of the pore, one may as well use MD.

Despite this, one can attempt to simulate dynamics using mean field theories by calculating a potential of mean force for a cargo. By placing a cargo particle a priori at a certain location along the central pore axis the free energy as a function of axial coordinate can be calculated. The free energy barrier to transport can then be estimated. The size of the free energy barrier facing a macromolecular cargo is related to the rate of transport of that cargo, with larger barriers generally meaning lower rates of transport.

This is the approach taken by Tagliazucchi et al., ${ }^{56}$ using a model of the nups similar to the one used by Peleg et $a l^{\mathbf{4 6}}$ Incorporated into this model are details of FG nup structure not included in previous mean-field simulations of NPC barrier organisation. Specific FG nups are included in terms of their amino acid sequences and approximate tethering position within the pore. Six categories of amino acids are considered, each with specific intermolecular interactions. Each amino acid interacts via a Lennard-Jones potential, with a binding energy of approximately $1.0 k_{\mathrm{B}} T$ for hydrophilic-hydrophilic interactions, $2.7 k_{\mathrm{B}} T$ for hydrophobic-hydrophobic interactions and $1.64 k_{\mathrm{B}} T$ for hydrophobic-hydrophilic interactions. As well as this extra level of detail, electrostatic interactions between different FG nups and the translocating cargo are also included.

These calculations suggest a homogeneous distribution of nups within the NPC. However, despite the homogeneous nup distribution, the calculations also show that the native amino acid sequence of the FG nups produces a highly inhomogeneous net charge distribution within the pore, with the central axis of the pore having a slightly positive net charge, which facilitates the transport of negatively charged cargoes, leading to a notable reduction in the free energy barrier presented to these particles. In fact, for the size of the translocating particles considered ( $5 \mathrm{~nm}$ ), the charge on the cargo plays a role in the translocation as significant as the hydrophobicity, with the free energy barrier of a charged, hydrophilic particle being of the same magnitude as the free energy barrier of a neutral hydrophobic one.

\section{Conclusions and outlook}

The understanding of the selectivity barrier of the NPC will require a combination of experimental and theoretical tools. Nevertheless, despite being an object of both biological and physical interest, the NPC has received relatively little attention from (bio-)physical modellers, probably due to the complexity of modelling such a large biological system. So far, different modelling approaches strongly suggest that an appreciation of the collective behaviour of nucleoporins is necessary to describe NPC functionality. As may be expected for a structurally flexible and interacting many-particle system, the exact choice of structural and physical/chemical parameters is crucial. Given 
the present experimental uncertainty on these parameters, ${ }^{57}$ it is not surprising that modelling approaches have not yet converged to a unified view on structure and function of the disordered nucleoporins in the central conduit. Rich physical behaviour has emerged from the models, which is also informative for the design of artificial nanopores, ${ }^{58,59}$ with useful technological applications. From the biological perspective, one may speculate whether the collective variability in the models is a signature of the structural flexibility of the central conduit that appears to be necessary for the selective gating of cargoes of widely varying sizes.

While the ensemble of various modelling approaches has been very informative about the range of structural behaviour that may be relevant in the NPC, the field is in need of simulations to offer guidance on the more pronounced aspects of the NPC selectivity barrier. A full molecular dynamics simulation of the NPC is unfeasible with current computational resources, but there is scope for further studies of smaller sections of the NPC along the lines of, ${ }^{33,44}$ especially if the geometry of the pore were taken into account. In general, it will be important to include physiological concentrations of Nuclear Transport Receptors (NTRs), because of their key role in transport and because recent experimental evidence suggests that they may play a significant part in the structure of the permeability barrier facing inert cargoes. ${ }^{\mathbf{6 0 , 6 1}}$

Some of the modelling approaches reviewed here can be used to estimate the size of the free energy barrier impeding macromolecular translocation, as seen in Tagliazucchi et al. ${ }^{\mathbf{5 6}}$ This, in turn, will enable a bridging between more detailed structural approaches and the diffusion equations mentioned above, treating the transport through the NPC as a Kramers problem. ${ }^{62}$ Different structural features could thus be correlated to experimentally determined transport rates, establishing the structure-function relation for the disordered nucleoporins in the central conduit and ultimately lead to a quantitative understanding of the selectivity barrier of the NPC.

\section{Acknowledgements}

The authors gratefully acknowledge Alan Lowe, Aksana Labokha and Aizhan Bestembayeva for discussions, and the Sackler Foundation, the UK Biotechnology and Biological Sciences Research Council (BB/G011729/1 and BB/J006254/1), and the Wellcome Trust $(083810 / \mathrm{Z} / 07 / \mathrm{Z})$ for funding.

\section{References}

1 A. Hoelz, E. W. Debler and G. Blobel, Annu. Rev. Biochem., 2011, 80, 613-643.

2 B. Hülsmann, A. A. Labokha and D. Görlich, Cell, 2012, 150, 738-751.

3 D. Stoffler, B. Feja, B. Fahrenkrog, J. Walz, D. Typke and U. Aebi, J. Mol. Biol., 2003, 328, 119-130.

4 M. Beck, F. Förster, M. Ecke, J. M. Plitzko, F. Melchior, G. Gerisch, W. Baumeister and O. Medalia, Science, 2004, 306, 1387-1390.
5 S. G. Brohawn, J. R. Partridge, J. R. Whittle and T. U. Schwartz, Structure, 2009, 17, 1156-1168.

6 D. Görlich and U. Kutay, Annu. Rev. Cell Dev. Biol., 1999, 15, 607-660.

7 D. Görlich, N. Panté, U. Kutay, U. Aebi and F. R. Bischoff, EMBO J., 1996, 15, 5584-5594.

8 E. Izaurralde, U. Kutay, C. von Kobbe, I. W. Mattaj and D. Görlich, EMBO J., 1997, 16, 6535-6547.

9 L. A. Strawn, T. Shen, N. Shulga, D. S. Goldfarb and S. R. Wente, Nat. Cell Biol., 2004, 6, 197-206.

10 M. K. Iovine, J. L. Watkins and S. R. Wente, J. Cell Biol., 1995, 131, 1699-1713.

11 M. Rexach and G. Blobel, Cell, 1995, 83, 683-692.

12 R. Bayliss, K. Ribbeck, D. Akin, H. M. Kent, C. M. Feldherr, D. Görlich and M. Stewart, J. Mol. Biol., 1999, 293, 579-593.

13 A. A. Labokha, S. Gradmann, S. Frey, B. B. Hülsmann, H. Urlaub, M. Baldus and D. Görlich, EMBO J., 2012, 32, 204-218.

14 K. Ribbeck and D. Görlich, EMBO J., 2001, 20, 1320-1330. 15 U. F. Greber and A. Fassati, Traffic, 2003, 4, 136-143.

16 S. Au and N. Panté, J. Struct. Biol., 2012, 177, 90-98.

17 W. Yang, J. Gelles and S. M. Musser, Proc. Natl. Acad. Sci. U. S. A., 2004, 101, 12887-12892.

18 U. Kubitscheck, D. Grünwald, A. Hoekstra, D. Rohleder, T. Kues, J. P. Siebrasse and R. Peters, J. Cell Biol., 2005, 168, 233-243.

19 B. Naim, D. Zbaida, S. Dagan, R. Kapon and Z. Reich, EMBO J., 2009, 28, 2697-2705.

20 R. Y. H. Lim, B. Fahrenkrog, J. Koser, K. Schwarz-Herion, J. Deng and U. Aebi, Science, 2007, 318, 640-643.

21 R. Peters, Traffic, 2005, 6, 421-427.

22 M. Tagliazucchi and I. Szleifer, Soft Matter, 2012, 8, 72927305.

23 S. P. Adiga and D. W. Brenner, J. Funct. Biomater., 2012, 3, 239-256.

24 F. Alber, S. Dokudovskaya, L. M. Veenhoff, W. Zhang, J. Kipper, D. Devos, A. Suprapto, O. Karni-Schmidt, R. Williams, B. T. Chait, A. Sali and M. P. Rout, Nature, 2007, 450, 695-701.

25 J. Yamada, J. L. Phillips, S. Patel, G. Goldfien, A. CalestagneMorelli, H. Huang, R. Reza, J. Acheson, V. V. Krishnan, S. Newsam, A. Gopinathan, E. Y. Lau, M. E. Colvin, V. N. Uversky and M. F. Rexach, Mol. Cell. Proteomics, 2010, 9, 2205-2224.

26 T. Bickel and R. Bruinsma, Biophys. J., 2002, 83, 3079-3087. 27 B. Nielsen, C. Jeppesen and J. H. Ipsen, J. Biol. Phys., 2006, 32, 465-472.

28 A. Zilman, S. Di Talia, B. T. Chait, M. P. Rout and M. O. Magnasco, PLoS Comput. Biol., 2007, 3, e125.

29 A. Zilman, S. Di Talia, T. Jovanovic-Talisman, B. T. Chait, M. P. Rout and M. O. Magnasco, PLoS Comput. Biol., 2010, 6, e1000804.

30 D. Chatterjee and B. J. Cherayil, J. Chem. Phys., 2011, 135, 155101.

31 V. Melnikov, Phys. Rep., 1991, 209, 1-71.

32 M. Karplus and J. A. McCammon, Nat. Struct. Mol. Biol., 2002, 9, 646-652. 
33 L. Miao and K. Schulten, Structure, 2009, 17, 449-459.

34 J. S. Mincer and S. M. Simon, Proc. Natl. Acad. Sci. U. S. A., 2011, 108, E351-E358.

35 R. Moussavi-Baygi, Y. Jamali, R. Karimi and M. R. K. Mofrad, Biophys. J., 2011, 100, 1410-1419.

36 T. Kustanovich and Y. Rabin, Biophys. J., 2004, 86, 20082016.

37 J. N. Israelachvili, Intermolecular and Surface Forces, Academic Press, 3rd edn, 2011.

38 S. Frey, R. P. Richter and D. Görlich, Science, 2006, 314, 815817.

39 K. Ribbeck and D. Görlich, EMBO J., 2002, 21, 26642671.

40 A. Kramer, I. Liashkovich, Y. Ludwig and V. Shahin, Pflügers Archiv European Journal of Physiology, 2008, 456, 155-162.

41 S. P. Adiga and D. W. Brenner, Nano Lett., 2005, 5, 25092514.

42 T. A. Isgro and K. Schulten, Structure, 2005, 13, 18691879.

43 T. A. Isgro and K. Schulten, J. Mol. Biol., 2007, 366, 330-345.

44 T. A. Isgro and K. Schulten, Structure, 2007, 15, 977-991.

45 L. Miao and K. Schulten, Biophys. J., 2010, 98, 1658-1667.

46 O. Peleg, M. Tagliazucchi, M. Kröger, Y. Rabin and I. Szleifer, ACS Nano, 2011, 5, 4737-4747.

47 R. H. Swendsen and J.-S. Wang, Phys. Rev. Lett., 1986, 57, 2607-2609.

48 A. Laio and F. L. Gervasio, Rep. Prog. Phys., 2008, 71, 126601. 49 R. Evans, Adv. Phys., 1979, 28, 143-200.
50 D. Osmanovic, J. Bailey, A. H. Harker, A. Fassati, B. W. Hoogenboom and I. J. Ford, Phys. Rev. E: Stat., Nonlinear, Soft Matter Phys., 2012, 85, 061917.

51 N. Metropolis, J. Chem. Phys., 1953, 21, 1087.

52 A. G. Koutsioubas, N. Spiliopoulos, D. L. Anastassopoulos, A. A. Vradis and C. Toprakcioglu, J. Chem. Phys., 2009, 131, 044901.

53 R. Wang, P. Virnau and K. Binder, Macromol. Theory Simul., 2010, 19, 258-268.

54 D. I. Dimitrov, A. Milchev and K. Binder, J. Chem. Phys., 2006, 125, 034905.

55 S. A. Egorov, A. Milchev, L. Klushin and K. Binder, Soft Matter, 2011, 7, 5669-5676.

56 M. Tagliazucchi, O. Peleg, M. Krger, Y. Rabin and I. Szleifer, Proc. Natl. Acad. Sci. U. S. A., 2013, 110, 3363-3368.

57 S. S. Patel, B. J. Belmont, J. M. Sante and M. F. Rexach, J. Chem. Phys., 2007, 129, 83-96.

58 T. Jovanovic-Talisman, J. Tetenbaum-Novatt, A. S. McKenney, A. Zilman, R. Peters, M. P. Rout and B. T. Chait, Nature, 2009, 457, 1023-1027.

59 S. W. Kowalczyk, L. Kapinos, T. R. Blosser, T. Magalhaes, P. van Nies, R. Y. H. Lim and C. Dekker, Nat. Nanotechnol., 2011, 6, 433-438.

60 J. Ma, A. Goryaynov, A. Sarma and W. Yang, Proc. Natl. Acad. Sci. U. S. A., 2012, 109, 7326-7331.

61 R. L. Schoch, L. E. Kapinos and R. Y. H. Lim, Proc. Natl. Acad. Sci. U. S. A., 2012, 109, 16911-16916.

62 H. Kramers, Physica, 1940, 7, 284-304. 